\title{
A SYMMETRY PROPERTY OF THE FRÉCHET DERIVATIVE
}

\author{
ROY MATHIAS \\ (Communicated by Lance W. Small)
}

\begin{abstract}
Let $A$ and $B$ be $n \times n$ matrices. We show that the matrix representing the linear transformation

$$
X \mapsto(A X B+B X A)^{T}
$$

(which is from the space of $n \times n$ matrices to itself) with respect to the usual basis is symmetric and show a similar symmetry property for the Fréchet derivative of a function $f(X)=\sum_{i=0}^{\infty} a_{i} X^{i}$ defined on the space of $n \times n$ matrices.
\end{abstract}

Let $M_{n}$ denote the space of complex $n \times n$ matrices. Given $X \in M_{n}$ we define $\operatorname{vec}(X)$ to be the vector in $C^{n^{2}}$ obtained by stacking the columns of $X$, i.e.,

$$
\operatorname{vec}(X)_{(j-1) n+i}=x_{i j}, \quad i, j=1, \ldots, n .
$$

Let $E_{i j} \in M_{n}$ denote the matrix with $i, j$ entry 1 and all other entries 0 . When we refer to the matrix representation of a linear transformation $L$ on $M_{n}$ we mean the representation with respect to the basis $\left\{E_{1,1}, E_{2,1}, \ldots\right.$, $\left.E_{n, 1}, E_{1,2}, \ldots, E_{n, n}\right\}$. With this notation the matrix that represents $L$ is the matrix $M$ such that $M \operatorname{vec}(X)=\operatorname{vex}(L(X))$ for all $X \in M_{n}$. Let $A \otimes B$ denote the Kronecker (or tensor) product of $A$ and $B$. Let $T_{n}$ denote the $n^{2} \times n^{2}$ permutation matrix such that $T_{n} \operatorname{vec}(X)=\operatorname{vec}\left(X^{T}\right)$ for all $X \in M_{n}$. Since $\left(X^{T}\right)^{T}=X$, it follows that $T_{n}=T_{n}^{-1}$. But because $T_{n}$ is a permutation matrix, we must also have $T_{n}^{-1}=T_{n}^{T}$, so $T_{n}=T_{n}^{-1}=T_{n}^{T}$.

We will make $M_{n}$ an inner product space with inner product $\langle A, B\rangle \equiv$ $\operatorname{tr} A B^{*}$. We say that a linear transformation $L$ on $M_{n}$ is Hermitian if

$$
\langle X, L(X)\rangle=\operatorname{tr}\left[X L(X)^{*}\right] \in \mathbb{R} \quad \forall X \in M_{n} .
$$

It is easy to check that if $M$, the matrix representing $L$, is real then $L$ is Hermitian if and only if $M=M^{T}$.

Let $f(z)=\sum_{i=0}^{\infty} a_{i} z^{i}$, where the series has radius of convergence $R$. Then for any $X \in M_{n}$ with spectral radius less than $R$ the Frechet derivative of $f$ at $X$ applied to $Z \in M_{n}$ can be shown to be

$$
L_{f}(X, Z)=\sum_{m=1}^{\infty} a_{i} \sum_{k=1}^{m} X^{k-1} Z X^{m-k} .
$$

Received by the editors July 29, 1992.

1991 Mathematics Subject Classification. Primary 15A04, 15A24, 15 A69.

Key words and phrases. Kronecker product, tensor product, Fréchet derivative, complex symmetric matrix. 
A derivation of this can be found at the beginning of $\S 2$ in [2].

We will show that the matrix representing the linear transformation $Z \mapsto$ $L_{f}(X, Z)^{T}$ is (possibly complex) symmetric, and we will show other results of this nature. This symmetry is exploited in an algorithm in [4].

First we will state some basic Kronecker product identities for $n \times n$ matrices $A, B, X$ :

$$
\begin{aligned}
\operatorname{vec}(A X B) & =B^{T} \otimes A \operatorname{vec}(X), \\
(A \otimes B)^{T} & =A^{T} \otimes B^{T}, \\
T_{n}(A \otimes B) & =(B \otimes A) T_{n} .
\end{aligned}
$$

These are Lemma 4.3.1, equation 4.2.2, and Corollary 4.3.10 of [1] respectively. Note that in [1] the matrix $T_{n}$ is denoted by $P(n, n)$.

We now combine these identities to obtain a simple result upon which our subsequent results are based.

Lemma 1. Let $A, B \in M_{n}$ be given. The matrix representation of the linear transformation

$$
L(X)=(A X B+B X A)^{T}
$$

is (possibly complex) symmetric. If in addition $A$ and $B$ are real then the linear transformation $L$ is Hermitian.

Proof. By (3) the matrix representation of $X \mapsto(A X B+B X A)$ is $B^{T} \otimes A+$ $A^{T} \otimes B$, so the matrix representation of $L$ is $M \equiv T_{n}\left(B^{T} \otimes A+A^{T} \otimes B\right)$. Let us show that $M^{T}=M$ :

$$
\begin{aligned}
M^{T} & =\left[T_{n}\left(B^{T} \otimes A+A^{T} \otimes B\right)\right]^{T}=\left(B^{T} \otimes A+A^{T} \otimes B\right)^{T} T_{n}^{T} \\
& =\left(B^{T} \otimes A\right)^{T} T_{n}+\left(A^{T} \otimes B\right)^{T} T_{n}=\left(B \otimes A^{T}\right) T_{n}+\left(A \otimes B^{T}\right) T_{n} \\
& =T_{n}\left(A^{T} \otimes B\right)+T_{n}\left(B^{T} \otimes A\right)=M .
\end{aligned}
$$

We have used $T_{n}^{T}=T_{n}$ for the third equality, (4) for the fourth, and (5) for the fifth.

If $A$ and $B$ are real then so is $M$. Since $M$ is real and symmetric, it follows that $L$ is Hermitian.

One can prove that if $A$ and $B$ are real then the linear transformation $L(X)=(A X B+B X A)^{T}$ is Hermitian without using Kronecker products.

$$
\begin{aligned}
\langle X, & \left.(A X B+B X A)^{T}\right\rangle=\operatorname{tr} X\left[(A X B+B X A)^{T}\right]^{*} \\
& =\operatorname{tr} X \overline{(A X B}+\operatorname{tr} X \overline{(B X A)}=\operatorname{tr} X A \bar{X} B+\operatorname{tr} X B \bar{X} A \\
& =\operatorname{tr} X A \bar{X} B+\operatorname{tr} \bar{X} A X B=\operatorname{tr} X A \bar{X} B+\overline{\operatorname{tr} X A \bar{X} B},
\end{aligned}
$$

which is real for all $X \in M_{n}$.

Theorem 2. Let $f(z)=\sum_{i=0}^{\infty} a_{i} z^{i}$, where the series has radius of convergence $R$. Let $X \in M_{n}$ have spectral radius less than $R$. Then the matrix representation of the linear transformation

$$
Z \mapsto\left[L_{f}(X, Z)\right]^{T}
$$

is (possibly complex) symmetric. If in addition $X$ is real and $a_{i}, i=1,2, \ldots$, are real then the linear transformation in (7) is Hermitian.

This result is true for a more general class of functions. We will discuss this generalization after Theorem 3. 
Proof. The linear transformation in (7) can be expressed as

$$
Z \mapsto \sum_{m=1}^{\infty} a_{i} \sum_{k=1}^{m}\left(X^{k-1} Z X^{m-k}+X^{m-k} Z X^{k-1}\right)^{T} / 2
$$

which is a sum of terms of the form $Z \mapsto(A Z B+B Z A)^{T}$. The result now follows from Lemma 1.

One can show that for the exponential function

$$
L_{\exp }(X, Z)=\int_{0}^{1} e^{t X} Z e^{(1-t) X} d t,
$$

for example, by substituting $e^{Y}=\sum_{0}^{\infty} Y^{k} / k$ ! , integrating term by term, and then comparing the result with (2). One way to estimate $L_{\exp }(X, Z)$ is to approximate the integral by the composite trapezoidal rule

$$
L_{\exp , T} \equiv \frac{1}{2^{k+1}}\left\{Z e^{X}+2 \sum_{j-1}^{2^{k}-1} e^{j X / 2^{k}} Z e^{\left(2^{k}-j\right) X / 2^{k}}+e^{X} Z\right\}
$$

or the composite Simpson's rule

$$
\begin{aligned}
L_{\exp , S} \equiv \frac{1}{6 \cdot 2^{k}}\{ & Z e^{X}+2 \sum_{j=1}^{2^{k}-1} e^{2 j X / 2^{k}} Z e^{\left(2^{k}-2 j\right) X / 2^{k}} \\
& \left.+4 \sum_{j=1}^{2^{k}-1} e^{(2 j-1) X / 2^{k}} Z e^{\left(2^{k}-2 j+1\right) X / 2^{k}}+e^{X} Z\right\} .
\end{aligned}
$$

It is useful to know that these approximations have the same symmetry property as $L_{\exp }(X, Z)$.

Theorem 3. Let $X \in M_{n}$. The matrix representations of the linear transformations

$$
Z \mapsto\left[L_{\exp , T}(X, Z)\right]^{T} \quad \text { and } \quad Z \mapsto\left[L_{\exp , S}(X, Z)\right]^{T}
$$

are (possibly) symmetric. If $X$ is real then the linear transformations in (8) are Hermitian.

Proof. The result for $L_{\text {exp }, T}$ follows from the formula

$$
\begin{aligned}
L_{\exp , T}(X, Z)=\frac{1}{2^{k+1}}\{ & \left(Z e^{X}+e^{X} Z\right) / 2 \\
& +2 \sum_{j=1}^{2^{k}-1}\left(e^{j X / 2^{k}} Z e^{\left(2^{k}-j\right) X / 2^{k}}+e^{\left(2^{k}-j\right) X / 2^{k}} Z e^{j X / 2^{k}}\right) / 2 \\
& \left.+\left(e^{X} Z+Z e^{X}\right) / 2\right\}
\end{aligned}
$$

and Lemma 1 . The result for $L_{\text {exp }, S}$ follows similarly.

Let $D$ be a domain in the complex plane, and let $f$ be analytic on $D$. The primary matrix function associated with $f$ is defined on the set of matrices with spectrum contained in $D$ as follows:

(a) if $A=S \operatorname{diag}\left(\lambda_{1}, \ldots, \lambda_{n}\right) S^{-1}$ then

$$
f(A)=S \operatorname{diag}\left(f\left(\lambda_{1}\right), \ldots, f\left(\lambda_{n}\right)\right) S^{-1},
$$

(b) if $A$ is not diagonalizable then define $f(A)$ by continuity. 
The analyticity of $f$ ensures that $f(A)$ is well defined. One can also define $f(A)$ via the Jordan form; this definition gives an explicit form even when $A$ is not diagonalizable. See $[1, \S 6.6]$ for this and a further discussion of primary matrix functions.

One can show that a primary matrix function is Fréchet differentiable at any $X$ with spectrum contained in $D$, so the Fréchet derivative is equal to the directional derivative. So by [1, Theorem $6.6 .14(3)]$

$$
L_{f}(X, Z)=\left.\frac{d}{d t} f(X+t Z)\right|_{t=0}=\left.\frac{d}{d t} p(X+t Z)\right|_{t=0}
$$

where $p$ is any polynomial such that if $\lambda$ is an eigenvalue of $X \oplus X$ of algebraic multiplicity $m$ then $p^{(i)}(\lambda)=f^{(i)}(\lambda), i=0,1, \ldots, m-1$. (Note that the restriction in [1, Theorem 6.6.14(3)] that $D$ be simply connected is not necessary.) Thus $L_{f}(X, Z)=L_{p}(X, Z)$, and since $p$ is just a polynomial it follows from Theorem 2 that the matrix representation of

$$
Z \mapsto\left[L_{f}(X, Z)\right]^{T}=\left[L_{p}(X, Z)\right]^{T}
$$

is (possibly complex) symmetric.

A primary matrix function that cannot be represented as a power series and for which one wants to compute the Fréchet derivative is the matrix sign function $\operatorname{sgn}(A)$ [3]. It corresponds to the function $f(z)=\operatorname{sign}(\operatorname{Re}(z))$ on $D=\{z: \operatorname{Re}(z) \neq 0\}$. An equivalent definition that is often used is

$$
\operatorname{sgn}(A)=S\left(\begin{array}{cc}
I & 0 \\
0 & -I
\end{array}\right) S^{-1},
$$

where $A=S\left(\begin{array}{ll}P & 0 \\ 0 & N\end{array}\right) S^{-1}$ and $P$ and $-N$ have spectrum in the open right-half plane.

\section{ACKNOWLEDGMENT}

Professor R. Horn pointed out that Theorem 2 is valid for primary matrix functions.

\section{REFERENCES}

1. R. A. Horn and C. R. Johnson, Topics in matrix analysis, Cambridge Univ. Press, New York, 1991.

2. C. Kenney and A. J. Laub, Conditions estimates for matrix functions, SIAM J. Matrix Anal. Appl. 10 (1989), 191-209.

3. __ Polar decomposition and matrix sign function condition estimates, SIAM J. Sci. Statist. Comput. 12 (1991), 488-504.

4. R. Mathias, Evaluating the Fréchet derivative of the matrix exponential, Numer. Math. 63 (1992), 213-226.

Department of Mathematics, College of William and Mary, Williamsburg, Virginia 23187

E-mail address: na.mathias@na-net.ornl.gov 